\title{
Therapeutic exosomes for various human diseases: Special issue of BMB Reports in 2022
}

Extracellular vesicles (EVs), especially exosomes, are cell-derived nanoparticles harboring various cellular components such as RNAs, lipids, and proteins for intercellular communication. Roles of EVs as intercellular communicators have been extensively studied in the last few decades, especially under various pathological conditions. Deciphering the message in EVs isolated from biological fluids of patients can provide valuable information not only for disease diagnosis, but also for disease monitoring or treatment responses. EVs are also attractive treatment modality and drug delivery system with favorable properties of biocompatibility, selective tropism, and stability. Stem cell-derived naïve EVs have been tested for their regenerative or immunomodulatory effects in numerous preclinical and clinical studies. This so-called "cell-free cell therapy" is supported by the idea that most therapeutic actions of conventional cell therapy are mediated by paracrine action of EVs released from stem cells. In that sense, immune cell-derived EVs are regarded as a reasonable option for cancer immunotherapy. Such therapeutic effect of EVs can be dramatically augmented by incorporating active pharmaceutical ingredients (APIs) to make engineered exosomes as "Trojan Horses". Biomimetic EVs or cell-derived nanovesicles can be generated through various physicochemical methos such as serial extrusion. They provide alternative options due to their high productivity and relatively easy purification. In this special issue, therapeutic applications of naïve or engineered EVs are discussed in various human diseases including cardiovascular diseases, renal disorders, neurological diseases, cancers, and infectious diseases focusing on COVID-19.

Acute and chronic kidney diseases are among major diseases targeted by EV therapeutics due to preferential renal distribution of EVs once they are systemically administered. Drs. SA Lee and TH Yoo summarize the current landscape of EV therapeutics focusing on naïve exosomes isolated from mesenchymal stem cells (MSCs) for acute kidney injury, chronic renal diseases, and graft failure after kidney transplantation. They further discuss pathological roles of EVs in kidney diseases through inflammatory, fibrotic, and thrombogenic properties. They also suggest potential therapeutic application of EV inhibition via suppression of production, secretion, and uptake by target cells. Lastly, the authors introduce the potential of engineered EVs loaded with APIs targeting NF- $\mathrm{KB}$ and TGF- $\beta$ pathways.

In the era of COVID-19 pandemic, it is important to prevent

https://doi.org/10.5483/BMBRep.2022.55.1.011

Received 27 December 2021 and treat hyperinflammatory responses observed in severe COVID-19 cases. Drs. H Choi and E-C Shin describe potential applications of EVs, naïve or engineered, for excessive inflammatory responses associated with severe COVID-19 cases. Along with conventional anti-inflammatory therapies, exosomes are now tried as a novel therapeutic modality for treating dysregulated inflammatory responses in COVID-19 based on promising results of exosomes in various inflammatory diseases and recent pilot clinical trials with COVID-19. The authors also discuss the potential of engineered $\mathrm{EV}$ s as the future direction of $\mathrm{EV}$ therapeutics for ameliorating inflammatory responses associated with systemic viral infections.

Because EVs are known to pass the blood brain barrier, EV therapeutics are being actively tested for various central nervous system (CNS) diseases. Drs. OY Bang and J-E Kim present convincing evidence for therapeutic potential of MSCs-derived naïve EVs to treat acute brain insults and chronic neurodegenerative diseases as an alternative of conventional stem cell therapy. In addition to promising efficacy, stem cell-derived EVs have better profiles of biocompatibility, immunogenicity, and safety than other conventional modalities including stem cell therapy. However, EV therapeutics also have disadvantages, including the lack of standardization for EV production, purification, and storage, complex characteristics of EV products and donor variation. For successful clinical translation, the authors emphasize the importance of scalable production along with quality control for regulatory authorities.

Drs. IS Kang and K Kwon describe the potential of naïve EVs derived from various cell sources such as MSCs, cardiac progenitor cells, induced pluripotent stem cells, and somatic cells from heart. Despite current EV therapeutics have huge potential, they also have many huddles before clinical translation. To overcome such obstacles, the authors propose various engineering strategies: enhanced production, cargo loading, surface modification, and reduced nonspecific clearance from system circulation.

Malignancies are the best studied therapeutic areas for EV therapeutics. Dr. Y Hong and I-S Kim describe the current understanding of adoptive cell therapy (ACT) and immune cell-derived exosomes and discuss limitations of ACT and opportunities for immune cell-derived exosomes as immune therapies. Since ACT of tumor-specific immune cells has proven clinical success in cancer treatment, EVs derived from immune cells offer sufficient therapeutic potential as substitutes for ACT due to their bio-functional cargo such as proteins and nucleic acids derived from their parental cells.

ISSN: 1976-670X (electronic edition)

Copyright (C) 2022 by the The Korean Society for Biochemistry and Molecular Biology

(c) This is an open-access article distributed under the terms of the Creative Commons Attribution Non-Commercial License (http://creativecommons.org/licenses/by-nc/4.0) which permits unrestricted non-commercial use, distribution, and reproduction in any medium, provided the original work is properly cited. 
Lastly, Dr. M-C Baek and colleagues from Kyungbook National University review the latest advances focusing on biological roles of immune cell-derived EVs for cancer therapy. The authors compare EVs derived from immune cells including dendritic cells (DCs), T cells, natural-killer (NK) cells, and macrophages for EV-based cancer therapy. To enhance the anti-cancer efficacy, the authors propose the potential of engineering EVs from cytokine-stimulated cells or antibody-decorated EVs.

In conclusion, EV therapy represents a promising modality for various human diseases ranging from viral infection to degenerative CNS diseases. Stem cell-derived naïve EVs are first shown to be an attractive alternative of conventional stem cell therapy, followed by further engineering of EVs for better effi- cacy, favorable distribution, and higher production yield. However, the lack of regulatory guideline, heterogeneity of EVs, quality control, and safety profiles are only subfractions of huddles to be addressed for further clinical translation of not only MSCsderived EVs, but also engineered EVs, even biomimetic EVs. Although continuous effects are needed to tackle all these obstacles, it is only a question of time that the current progress of EV therapeutics will lead to successful translation.

Chulhee Choi, M.D., Ph.D. ${ }^{1,2}$

${ }^{1}$ ILIAS Biologics Inc., Daejeon 34014, ${ }^{2}$ Department of Bio and Brain Engineering, KAIST, Daejeon 34141, Korea

E-mail: cchoi@iliasbio.com 